\title{
221 Eos: a remnant of a partially differentiated parent body?
}

\author{
T. Mothé-Diniz and J. M. Carvano
}

\author{
Observatoire de Paris/Meudon (LESIA), 15 place Jules Janssen, 92150 Meudon Cedex, France \\ e-mail: thais.mothe@obspm.fr
}

Received 1 June 2005 / Accepted 30 June 2006

\section{ABSTRACT}

\begin{abstract}
The asteroid 221 Eos and the objects belonging to its associated family - most of which belong to the K taxonomic class, have been traditionally associated with CO3/CV3 meteorites. The 52 color spectrum and the SMASSIR spectrum of 221 Eos in the NIR region, combined with the SMASSII spectrum in the visible, has now been compared to the whole RELAB meteorites database updated in July 2003. In this paper we present the first observational evidence that 221 Eos is analogous to a differentiated meteorite: the anomalous stone Divnoe. This link suggests that the the parent body of the Eos dynamical family was partially differentiated, with a thermal history quite different from what is currently accepted.
\end{abstract}

Key words. minor planets, asteroids

\section{Introduction}

The asteroid 221 Eos is the largest member of the Eos dynamical family, which is located on the outer part of main asteroid belt. Since the work of Bell (1988), the asteroid 221 Eos has been associated with $\mathrm{CO} / \mathrm{CV}$ anhydrous meteorites. In that work, Bell compared the spectrum of Eos in the NIR $(0.33-2.5 \mu \mathrm{m})$ with the available meteorite spectral data, and noted that the spectra and albedo of some Eos family objects were best matched by $\mathrm{CV}$ and $\mathrm{CO}$ chondrites. Latter, Doressoundiram et al. (1998) reinforced the association of the Eos family with $\mathrm{CO} / \mathrm{CV}$ chondrites, by showing similarities between the spectra of Eos family members and these types of meteorites in the visible range $(0.48-0.92 \mu \mathrm{m})$. The authors compared the spectra of the family members with the meteorites measured by Gaffey (1976). The asteroid Eos, however, was not observed in their survey of the family. Recently, Burbine et al. (2001) compared the NIR $(0.44-1.65 \mu \mathrm{m})$ spectrum of 221 Eos with a number of $\mathrm{CO} 3 / \mathrm{CV} 3$ meteorites and the meteorite Warrenton was found to be the most similar to Eos among the carbonaceous chondrites.

Since these works, however, the number of meteorite spectra in public databases have steadly increased. To perform an unbiased comparison of asteroid spectra with an up-to-date meteorite spectral database, we have compared the spectra of 221 Eos with 1478 meteorite spectra. The resulting best meteorite analog for Eos is not a $\mathrm{CO} / \mathrm{CV}$ meteorite, but the anomalous achondrite Divnoe. In the next section we describe how the comparison was done and discuss the spectral similarities between Eos, Divnoe and the previous best analog to Eos, the CO3 Warrenton. In Sect. 3 we discuss the problems and implications of a genetic link between Eos and Divnoe, and in Sect. 4 we summarize the main conclusions of this paper.

\section{Results}

For the comparison with the meteorite data we have used two spectra of Eos: (1) the 52-color (Bell et al. 1988) in the NIR $(0.82-2.46 \mu \mathrm{m})$ combined with the SMASSII (Bus $\&$ Binzel 2002) spectrum in the visible $(0.43-0.92 \mu \mathrm{m})$; and (2) the SMASSIR (Burbine \& Binzel 2002) in the NIR $(0.44-1.65 \mu \mathrm{m})$ also combined with the SMASSII spectrum. The composite Visible-NIR (VNIR) spectra have been obtained by requiring that the reflectance of the NIR portion matched the visible spectrum at $0.8 \mu \mathrm{m}$. The resulting spectra were normalized to unity at $0.55 \mu \mathrm{m}$ by convention.

The meteorite spectra were obtained from the RELAB public database (Pieters \& Hiroi 2004) updated on 31th July, 2003, which contains the spectra of 802 meteorite samples, with more than one spectrum for some of the samples, corresponding to different grain sizes and/or observation geometry. In order to perform an automated first order search, the meteorite and the asteroid spectra were interpolated to a common wavelength sampling in the spectral range covered by the asteroid spectra. The dispersion between the resampled asteroid and meteorite spectra was then calculated for each spectrum in the database and sorted into ascending order of dispersion. Plots of the asteroids and meteorite spectra made according to the resulting list were then visually inspected until it was obvious that no good match could be found with higher dispersion values. For Eos, the Divnoe meteorite and seveal $\mathrm{CO} / \mathrm{CV}$ meteorites appear with low dispersion values in the list, but visual inspection points to Divnoe, sample MB-CMP-015-D, as the best spectrum analog. Among the $\mathrm{CO} / \mathrm{CV}$ meteorites analysed, the best analogous was found to be the CO3 Warrenton, which agrees with the work of Burbine \& Binzel (2002). 


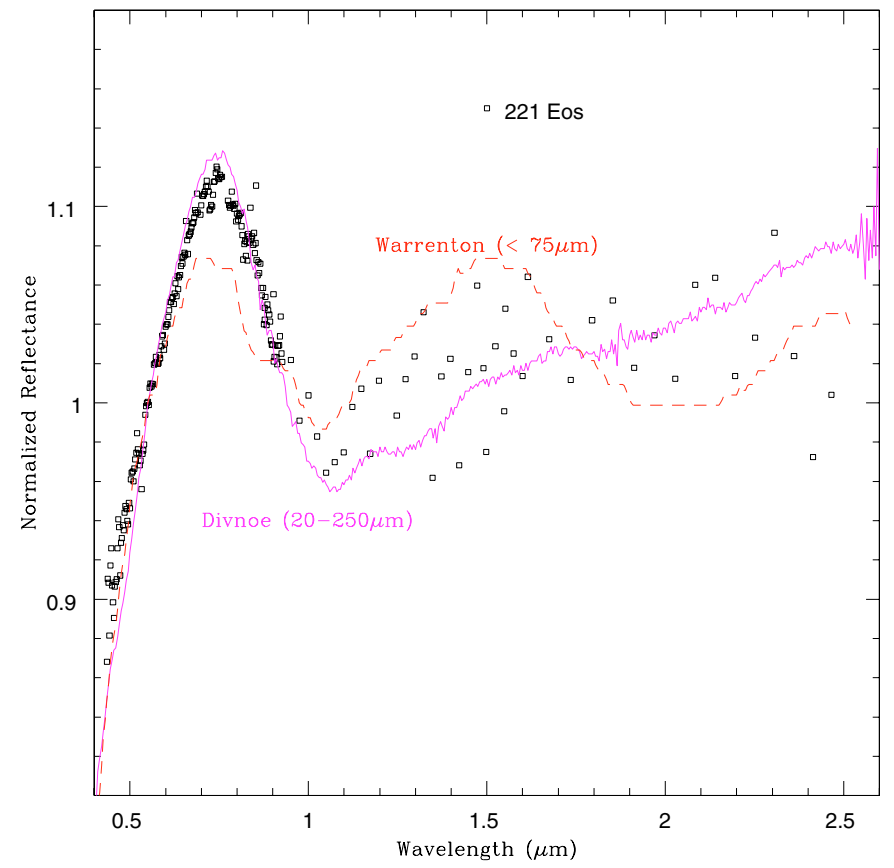

Fig. 1. Reflectance spectra for 221 Eos (squares) versus spectra of $\mathrm{CO} 3$ chondrite Warrenton and the anomalous stone Divnoe. The visible part of the Eos spectrum is from SMASS II (Bus \& Binzel 2002) and and its near-infrared spectrum is from 52-color data (Bell et al. 1988). All spectra are normalized to unity at $0.55 \mu \mathrm{m}$. In parenthesis we indicate the grainsize of the sample.

In Fig. 1, the composite 52-color+SMASS spectrum of Eos is compared with the spectrum of the meteorites Warrenton and Divnoe. The same plot but with the composite spectrum obtained with the SMASSIR spectrum is shown in Fig. 2. The main similarities between the spectrum of Divnoe and Eos that lead us to propose Divnoe as the best meteoritical analog are the following:

1. Divnoe is a perfect match in the visible region. In particular, the position of the maximum of reflectance in the asteroid spectra agrees perfectly with that of the meteorite. In contrast, the maximum of reflectance of Warrenton appears shifted to lower wavelengths with respect to the asteroid. Also, the spectrum of Warrenton shows an absorption band around $0.85 \mu \mathrm{m}$, which is not seen in the asteroid spectrum.

2. The positions of the minimum of reflectance of the spectra of Divnoe and Eos both occur around $1.08 \mu \mathrm{m}$. This can be more cleary seen in Fig. 2. On the other hand, the minimum of reflectance of Warrenton again seems to be shifted to a lower wavelength.

3. The spectrum of Warrenton presents a band around $2.0 \mu \mathrm{m}$, which does not seems to be present in the Eos spectra. This is not obvious from Fig. 1 because of the noise of the 52-color spectrum in this region, but the presence of this band in the Warrenton spectrum produces a maximum around $1.5 \mu \mathrm{m}$ that is clearly not present in the SMASSIR spectrum (Fig. 2). In contrast, the spectrum of Divnoe follows closely the behaviour the spectrum of Eos in this region.

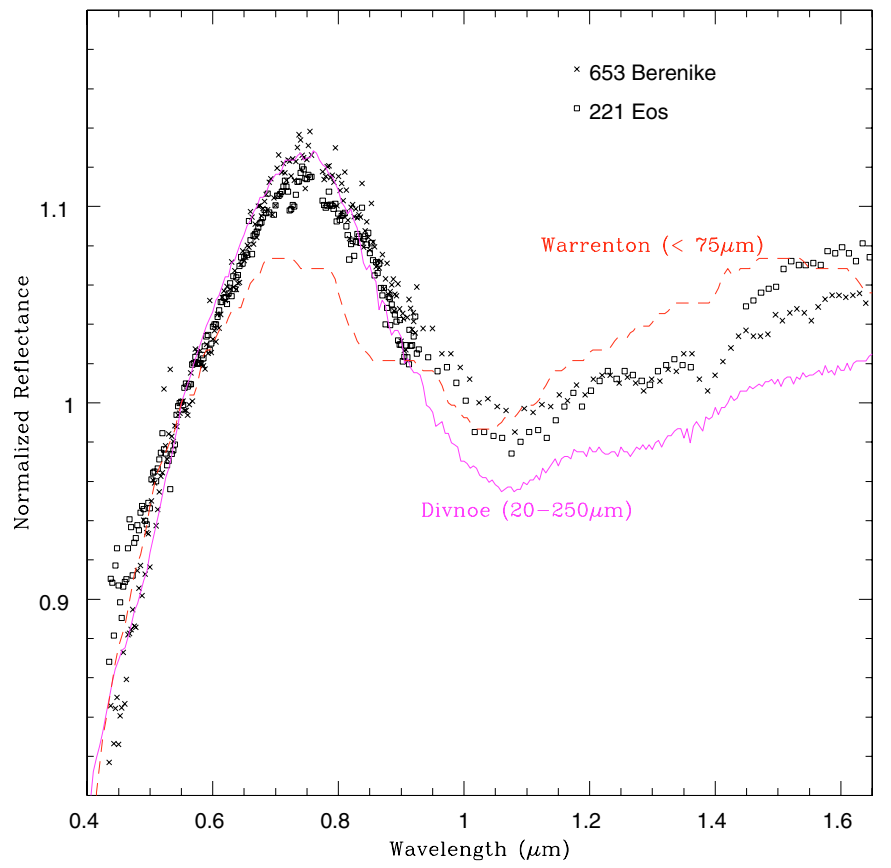

Fig. 2. Reflectance spectra for 221 Eos (squares) and 653 Berenike (crosses) versus spectra of the $\mathrm{CO} 3$ chondrite Warrenton and the anomalous stone Divnoe. The visible part of the Eos and Berenike spectra are from SMASS II (Bus \& Binzel 2002) and and the nearinfrared spectra are from SMASSIR (Burbine \& Binzel 2002). All spectra are normalized to unity at $0.55 \mu \mathrm{m}$. In parenthesis we indicate the grainsize of the sample.

\section{Discussion}

The spectral similarities between Divnoe and Eos shown in the previous section suggest a genetic link between the meteorite and the asteroid. In order to assess the plausibility of this link, points like the albedo compatibility between the asteroid and meteorite, the existence of suitable transport mechanism and the implications for the Eos family must be adressed. Before we do so, we briefly summarise what is presently known about this meteorite.

The Divnoe meteorite, an olivine-rich achondrite with high contents of troilite and metal, was found in Russia in 1981 with a total weight of $12.7 \mathrm{~kg}$ (Petaev et al. 1994, and references therein). It is considered an ungrouped primitive achondrite, since it has a mineral composition close to that of ordinary chondrites but presents an achondrite texture and also shows anomalous abundances of rare earth elements (Petaev et al. 1994; Shukolyukov et al. 1995). Its relations to other meteorite groups are unclear. In isotopic composition of O, Divnoe is close to (but distinct from) the $\mathrm{IAB} /$ winonaite group, to the HED-meteorites and to brachinites (Petaev et al. 1994). On the basis of Ar- and He-isotopic composition, a link to diogenites (but not to eucrites or howardites) was sugested by Shukolyukov et al. (1995). However Weigel et al. (1996) pointed out that the Xe-isotopic composition of Divnoe is inconsistent with diogenites and suggested instead that it may be related to brachinites based on Xe-isotopic composition and similarities in bulk chemistry. The isotopic ratios on Divnoe suggest an early formation of its parent-body (K-Ar age of 
around 4.6 Byr) and that the meteorite broke off from its parent-body 17.2 Myr ago (Shukolyukov et al. 1995). The bi-directional reflectance spectra in the $0.32-0.7 \mu \mathrm{m}$ range of a sample of Divnoe was obtained by Carlé Pieters in November, 1990, in the RELAB facility at Brown University, and was made publicly availabe in December, 2000.

It is usual in the literature to compare the visible geometric albedo of asteroids to the reflectance of the meteorite at $0.55 \mu \mathrm{m}$, even if it is known that these two quantities may not be directly comparable (e.g. Carvano et al. 2003; Burbine et al. 2001). The IRAS geometric visual albedo of Eos ( $p_{v}=$ $0.14 \pm 0.01$, Tedesco et al. 2002) is somewhat closer to the reflectance at $0.55 \mu \mathrm{m}$ of Warrenton $(0.17)$ than to that of Divnoe (0.08), but the difference is not enough to preclude a link between Eos and Divnoe. Moreover, the IRAS albedo determination is somewhat model-dependent and tends to overestimate the albedo of colder, outer main belt objects (Spencer et al. 1989).

Concerning the transport mechanism, di Martino et al. (1997) modeled the evolution of particles ejected from the Eos family. They reported a very small delivery efficiency: only $1 \%$ of the particles achieved an Earth-crossing orbit, in a minimum time of $50 \mathrm{Myr}$. Similar calculations performed by Zappala et al. (1998) suggested that the most likely transport times from the Eos region to Earth-crossing orbits are in the range 110-140 Myr. These transport times seem incompatible with the exposure age of Divnoe (17.2 Myr) but also with the exposure age of 34 Myr estimated for Warrenton (Scherer $\&$ Schultz 2000). One way to reconcile the exposure age of Divnoe with those of transport times is to suppose that Divnoe originated through a collisional cascade process (di Martino et al. 1997). It is also noteworthy that neither di Martino et al. (1997) nor Zappala et al. (1998) included the Yarkovsky effect in their simmulations, which could contribute to considerably shorten meteorite transport times (Bottke et al. 2002, and references therein). Recently, Vokrouhlický et al. (2005) developed a detailed theory of Yarkovsky evolution for the Eos family. This work suggests that drifting produced by the Yarkovsky effect could inject fragments into the $9 / 4$ and $7 / 3$ mean motion resonances, decreasing the transport times. Considering the delivery efficiency, di Martino et al. (1997) concluded that only $2 \%$ of the $\mathrm{CO} / \mathrm{CV}$ chondrites could originate at the Eos region. In contrast, Divnoe is a unique meteorite which may be related to the also rare brachinites (Weigel et al. 1996), which seems more consistent with an inefficient delivery mechanism.

Finally, the association between Eos and Divnoe implies a very different thermal history for the parent body for the Eos family than if a $\mathrm{CO} / \mathrm{CV}$ composition is assumed. The parent body of Divnoe suffered partial melting at $\sim 1300{ }^{\circ} \mathrm{C}$, followed by a catastrophic removal of the liquid portion of the melt and recrystalization (Petaev et al. 1994). On the other hand, the $\mathrm{CO} / \mathrm{CV}$ meteorites experienced metamorphism at temperatures inferior to $\sim 950{ }^{\circ} \mathrm{C}$. Therefore, the breakup of the parent-body of the Divnoe meteorite should produce a more mineralogicaly diverse family than the breakup of a $\mathrm{CO} / \mathrm{CV}$ parent-body. Indeed, the Eos family presents considerable spectral diversity (Doressoundiram et al. 1998; Mothé-Diniz et al. 2005), which might be consistent with the break-up of a partially differentiated parent-body, even if the spectral diversity of the $\mathrm{CO} / \mathrm{CV}$ could account for at least some of the spectral dispersion seen in the family (Mothé-Diniz et al. 2005). Another Eos family member, 653 Berenike, has a spectrum that is almost identical to the spectrum of 221 Eos (Burbine et al. 2001), and which is also a very good match to the spectrum of Divnoe.

\section{Conclusions}

Here we have shown that, among all meteorites in the RELAB database, the spectrum of Divnoe is the best analog to the available VNIR spectra of 221 Eos. As in the previous meteoritc match to Eos, this link is based on a qualitative spectral comparison. In order to confirm this relationship, it is necessary to perform a detailed mineralogic analysis of high signal-tonoiseVNIR spectra of 221 Eos and of members of the Eos dynamical family. Such an analys is under way.

Acknowledgements. This work has been supported by the Conselho Nacional de Desenvolvimento Científico e Tecnológico $\mathrm{CNPq} / \mathrm{Brasil}$. This research utilizes spectra acquired with the NASA RELAB facility at Brown University. We thank Dr. D. Vokrouhlický for the helpful review of the manuscript.

\section{References}

Bell, J. F. 1988, Meteoritics, 23, 256

Bell, J. F., Owensby, P. D., Hawke, B. R., \& Gaffey, M. J. 1988, in Lunar and Planetary Institute Conference Abstracts, 57

Bottke, W. F., Vokrouhlický, D., Rubincam, D. P., \& Broz, M. 2002, Asteroids III, 395

Burbine, T. H., \& Binzel, R. P. 2002, Icarus, 159, 468

Burbine, T. H., Binzel, R. P., Bus, S. J., \& Clark, B. E. 2001, Meteor. Planet. Sci., 36, 245

Bus, S. J., \& Binzel, R. P. 2002, Icarus, 158, 106

Carvano, J. M., Mothé-Diniz, T., \& Lazzaro, D. 2003, Icarus, 161, 356

di Martino, M., Migliorini, F., Cellino, A., \& Zappala, V. 1997, Meteor. Planet. Sci., 32, 35

Doressoundiram, A., Barucci, M. A., Fulchignoni, M., \& Florczak, M. 1998, Icarus, 131, 15

Gaffey, M. J. 1976, J. Geophys. Res., 81, 905

Mothé-Diniz, T., Roig, F., \& Carvano, J. M. 2005, Icarus, 174, 54

Petaev, M. I., Barsukova, L. D., Lipschutz, M. E., et al. 1994, Meteoritics, 29, 182

Pieters, C. M., \& Hiroi, T. 2004, in Lunar and Planetary Institute Conference Abstracts, 1720

Scherer, P., \& Schultz, L. 2000, Meteor. Planet. Sci., 35, 145

Shukolyukov, Y. A., Assonov, S. S., Smoliar, M. I., \& Kolesnikov, E. M. 1995, Meteoritics, 30, 654

Spencer, J. R., Lebofsky, L. A., \& Sykes, M. V. 1989, Icarus, 78, 337 Tedesco, E. F., Noah, P. V., Noah, M., \& Price, S. D. 2002, AJ, 123, 1056

Vokrouhlický, D., Brož, M., Morbidelli, A., et al. 2005, Icarus, submitted

Weigel, A., Eugster, O., Koeberl, C., \& Krahenbuhl, U. 1996, in Lunar and Planetary Institute Conference Abstracts, 1403

Zappala, V., Cellino, A., Gladman, B. J., Manley, S., \& Migliorini, F. 1998, Icarus, 134, 176 